\title{
Resultados a corto y mediano plazo del tratamiento de la obstrucción al tracto de salida del ventrículo izquierdo mediante miectomía videoasistida
}

\author{
Federico Paredes ${ }^{1}$, Sergio Cánovas1, Rafael García-Fuster ${ }^{1}$, Óscar Gil ${ }^{1}$, Fernando Hornero ${ }^{1}$, Elio \\ Martín ${ }^{1}$, Armando Mena ${ }^{1}$, Bruno Bochard ${ }^{2}$, Juan Martínez-León ${ }^{1}$.
}

\author{
Instituto Cardiovascular \\ 1 Servicio de Cirugía Cardíaca. \\ 2 Servicio de Cardiología \\ Hospital General Universitario de Valencia. \\ Valencia-España.
}

Objetivo: Describir nuestra experiencia y evaluar los resultados a corto y mediano plazo de la miectomía videoasistida en el tratamiento de la obstrucción del tracto de salida del ventrículo izquierdo (OTSVI) en pacientes con miocardiopatía hipertrófica.

Materiales y métodos: 52 pacientes con edad media de 56,2 (rango 12 - 83) y Euroscore de riesgo de 4,1 $\pm 1,92$ con diagnóstico de OTSVI fueron intervenidos de manera consecutiva en un mismo centro mediante miectomía videoasistida. Se realizó seguimiento clínico y ecocardiográfico con controles al mes y al año del postoperatorio.

Resultados: Entre las patologías asociadas encontramos 11 pacientes con valvulopatía aórtica, 2 con valvulopatía mitral, 3 con cardiopatía isquémica, 1 con aneurisma de aorta ascendente y 1 con foramen oval permeable resueltos en el mismo acto quirúrgico. La mortalidad hospitalaria global fue de 5,8\% (3 pacientes). En el momento de la intervención, 8 (15,4\%) se encontraban en clase funcional II de la NYHA, 42
$(80,8 \%)$ en clase III y $2(3,8 \%)$ en clase IV. El gradiente máximo subaórtico disminuyó de $80,7 \mathrm{mmHg}$ $\pm 29,43$ en el preoperatorio a $19,0 \mathrm{mmHg} \pm 15,57$ $(p<0,001)$ en el postoperatorio inmediato, manteniéndose en $14,6 \mathrm{mmHg} \pm 8,88$ al mes $(\mathrm{p}<0,001$ en relación al preoperatorio) y al año en $13,9 \mathrm{mmHg} \pm$ 7,69 ( $\mathrm{p}<0,001$ en relación al preoperatorio). Además, se registró una disminución del grosor del tabique interventricular en diástole de $19,4 \mathrm{~mm} \pm 3,78$ en el preoperatorio a $12,9 \mathrm{~mm} \pm 2,35(\mathrm{p}<0,001)$ en el postoperatorio. Todos los pacientes se encontraban en clase funcional I-II al final del seguimiento.

Conclusión: Los resultados demuestran que la miectomía videoasistida es un tratamiento seguro para la OTSVI con el que se obtienen resultados favorables a corto y mediano plazo, tanto en parámetros clínicos, como ecocardiográficos.

Palabras claves: Miocardiopatía hipertrófica obstructiva, miectomía septal, miectomía video asistida. 


\section{Video assisted myectomy for relief of left ventricular tract obstruction in patients with Obstructive Hypertrophic Cardiomyopathy}

Aim: To report a clinical experience and to evaluate early and mid term results of video assisted myectomy for relief of left ventricular tract obstruction (LVOTO) in patients with Obstructive Hypertrophic Cardiomyopathy.

Methods: 52 patients with Obstructive Cardiomyopathy and a mean age 56.2 years $(12-83)$ carrying a Euro score risk of $4.1 \pm$ (SD 1.92), were consecutively operated on in a single center. Relief of LVOTO was performed with video assisted myectomy. Clinical and echocardiographic follow up to 1 year postoperatively was carried out.

Results: Apart from the Obstructive Cardiomyopathy, 11 patients had aortic valve disease, 2 mitral valve disease, 3 ischemic heart disease, 1 an ascending aortic aneurysm and 1 a patent foramen ovale. All these lesions were surgically repaired in the same surgical act. In hospital mortality was $5.8 \%$ (3 patients). Pre-operatively $15.4 \%$ of patients were in NYHA Class II, $80.8 \%$ in Class III and 3.8\% in Class IV. After surgery peak sub aortic gradient decreased from $80.7 \pm 29.43 \mathrm{mmHg}$ to $19.0 \pm 15.57(\mathrm{p}<0.001)$. Corresponding values were $14.6 \pm 8.88$ at 1 month and $13.9 \pm 7.69$ at 1 year post operatively. Interventricular septal thickness in diastole decreased from $19.4 \pm 3.78 \mathrm{~mm}$ to $12.9 \pm 2.35 \mathrm{~mm}$ after surgery $(\mathrm{p}<0.001)$. All patients were Class I or II at the end of follow up.

Conclusion: Video assisted myectomy is safe and effective for relief of LVOTO in patients with hypertrophic cardiomyopathy. Good results are maintained one year after surgery.

Keywords: Obstructive Hypertrophic Cardiomyopathy, Cardiac Surgery, video assisted myectomy

\section{Introducción}

La obstrucción al tracto de salida del ventrículo izquierdo (OTSVI) es una entidad compleja debido a la variedad morfológica que pueden presentar las lesiones causantes, motivo por el cual constituye un desafío para los cirujanos. ${ }^{1}$ Dentro del abanico de causas nos encontramos desde la presencia de una simple membrana subaórtica que produce una discreta estenosis, pasando por grados más complejos, con tejido fibroso que se extiende hasta la válvula aórtica, impidiendo la apertura correcta de la misma, creando un gradiente subaórtico importante. ${ }^{2,3,4}$ Además, alteraciones del aparato subvalvular mitral pueden ocasionar una obstrucción persistente al tracto de salida del ventrículo izquierdo. ${ }^{5}$

La miocardiopatía hipertrófica es un desorden genético que afecta aproximadamente al $0,2 \%$ de la población. ${ }^{6}$ Esta entidad puede resultar en una hipertrofia septal extrema produciendo una OTSVI. ${ }^{7}$ Se caracteriza por una hipertrofia del ventrículo izquierdo asociada a cavidades ventriculares normales (sin dilatación) en ausencia de otra enfermedad cardíaca o sistémica que por si sola sea capaz de producir ese grado de hipertrofia. ${ }^{6,8,9,10}$ Clínicamente, la miocardiopatía hipertrófica se reconoce habitualmente por un grosor máximo de la pared del ventrículo izquierdo $\geq 15 \mathrm{~mm}^{11}$

Hemodinámicamente, se considera obstrucción dinámica al tracto de salida del ventrículo izquierdo un gradiente de salida $\geq 30 \mathrm{mmHg}$ en reposo. Existen pacientes que no presentan OTSVI en reposo, sin embargo, en ciertas condiciones como el ejercicio, las maniobras de Valsalva 
o durante la provocación farmacológica, el gradiente de salida puede aumentar hasta producir una obstrucción dinámica. ${ }^{12,13}$

El tratamiento quirúrgico de esta patología se reserva para los pacientes que presentan obstrucción dinámica al tracto de salida, se encuentran sintomáticos y no responden al tratamiento médico.

Dado que se trata de una patología poco habitual, el propósito de este trabajo es mostrar la experiencia de nuestro centro en el tratamiento quirúrgico de la OTSVI mediante miectomía video asistida, describiendo la misma, así como los resultados obtenidos.

\section{Materiales y métodos}

Se trata de un estudio retrospectivo con datos obtenidos en un sólo centro, extraídos de la base informática del servicio. Un total de 52 pacientes con edad media de 56,2 años (rango 12-83) y EuroScore Logístico de 4,1 $\pm 1,19$, todos con diagnóstico de OTSVI por hipertrofia muscular fueron intervenidos de manera consecutiva mediante miectomía videoasistida en el período comprendido entre enero del 2005 y junio del 2012. Todos los pacientes fueron sometidos a seguimiento tanto clínico como ecocardiográfico con controles realizados en el postoperatorio inmediato, al mes y al año de la intervención.

\section{Técnica quirúrgica.}

Se realizó ecocardiografía transesofágica (ETE) intraoperatoria previa a la intervención para cuantificar la obstrucción y medir tanto el gradiente como el grosor del tabique. En 48 pacientes se utilizó la esternotomía media longitudinal como abordaje y en 4 se practicó miniesternotomía en "J" invertida (abordaje mínimamente invasivo). Para establecer la circulación extracorpórea (CEC) se utilizó canulación central estándar en todos los pacientes con idéntico régimen de cardioplejia hemática anterógrada fría para establecer la parada cardíaca. Una vez realizada la aortotomía transversa se procedió a exponer el tracto de salida mediante el pliegue de los velos aórticos con suturas de prolene 7/0 en los casos en los que sería conservada la válvula y mediante resección de los velos en los casos en que sería reemplazada. Posteriormente, bajo visión directa y, además, mediante videoasistencia con una óptica de $30^{\circ}$ introducida al campo por la misma esternotomía, se procedió a resecar el tracto de salida con bisturí eléctrico de asa. Posterior al desclampaje y salida de CEC se procedió a comprobar el resultado mediante ecocardiograma trans esofágico (ETE).

\section{Análisis estadístico.}

Los datos fueron analizados mediante el paquete estadístico SPSS (versión 19.0). Estos datos son expresados como frecuencias, medias con desviación estándar o medias con rango.

Para los análisis estadísticos cuantitativos fue utilizado el test U de Mann-Whitney. Las diferencias fueron consideradas significativas cuando $\mathrm{p}$ fue $<0,05$.

El trabajo fue aprobado por el Comité de Ética del Instituto Cardiovascular.

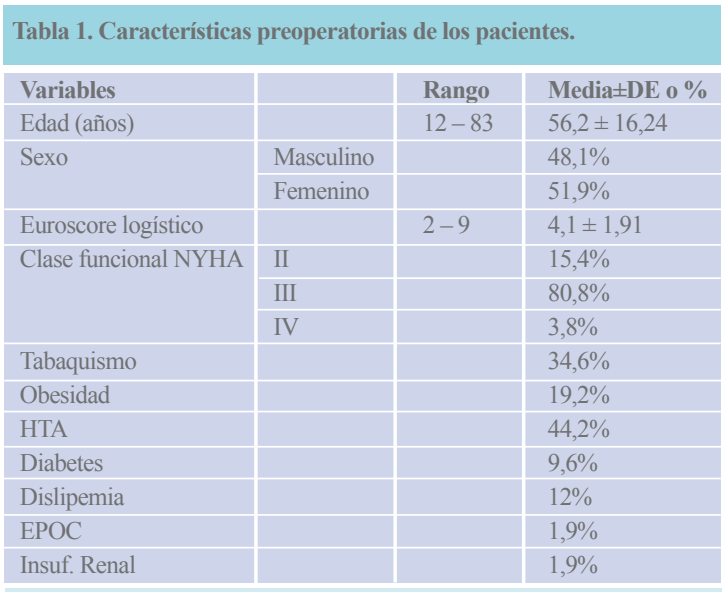

EPOC: Enfermedad pulmonar obstructiva crónica; NYHA: New York Heart Asociation; Insuf. Renal: insuficiencia renal

\section{Resultados}

Las características preoperatorias de los 52 pacientes se describen en la Tabla 1 . El rango de edad fue de 12 a 83 años (media 56,2 $\pm 16,24)$. Se encontró un leve predominio de pacientes de sexo femenino, $51,9 \%$. El $80 \%$ de los pacientes se encontraba en clase funcional III de la NYHA previo a la intervención. Todos presentaban síntomas refractarios al tratamiento médico. Entre las patologías asociadas encontramos 11 pacientes con valvulopatía aórtica, 2 con valvulopatía mitral, 3 con cardiopatía isquémica, 1 con un aneurisma de aorta ascendente y 1 con un foramen oval permeable, todas resueltas en el mismo acto quirúrgico (Tabla 2). La mortalidad global fue 5,8\% (3 pacientes), dos casos fueron de causa cardíaca (shock cardiogénico) y un caso de sepsis de origen respiratorio más un cuadro de distress como parte probable de un síndrome post trans- 
Tabla 2. Patologías asociadas a la OTSVI tratadas quirúrgicamente.

Proc. asociados

\begin{tabular}{|l|l|}
\hline Bypass aorto-coronario & $5,6 \%(3)$ \\
\hline Cirugía aórtica & $1,9 \%(1)$ \\
\hline Cierre Foramen Oval & $1,9 \%(1)$ \\
\hline Cirugía valvular & $25 \%(13)$ \\
\hline Mitral & $3,84 \%(2)$ \\
\hline Aórtica & $21,15 \%(11)$ \\
\hline
\end{tabular}

fusional. Los datos clínicos y ecocardiográficos se obtuvieron del 100\% de los pacientes en el preoperatorio, del $100 \%$ en el intraoperatorio inmediato a la salida de CEC, $94,2 \%$ (49 pacientes) al mes (perdidos 3 pacientes correspondientes a la mortalidad encontrada) y $80 \%$ (45 pacientes) al año (4 pacientes intervenidos hacía menos de 1 año del análisis de los datos).

\section{Seguimiento ecocardiográfico (Tabla 3) (Figura 1).}

\begin{tabular}{|c|c|c|c|c|}
\hline \multicolumn{5}{|l|}{ Datos ecocardiográficos } \\
\hline Número pacientes & $52(100 \%)$ & $52(100 \%)$ & $49(94,2 \%)$ & $45(80 \%)$ \\
\hline Tiempo de seguimiento & Pre Qx & $\begin{array}{l}\text { Post } \\
\text { Qx Inmediato }\end{array}$ & $1 \mathrm{mes}$ & 1 año \\
\hline $\begin{array}{l}\text { Tabique Interventricular } \\
\text { en Diástole (mm) }\end{array}$ & $19,4 \pm 3,78$ & $13,6 \pm 2,21$ & $13,0 \pm 2,17$ & $12,9 \pm 2,34$ \\
\hline $\begin{array}{l}\text { Tabique interventricular } \\
\text { en Sístole (mm) }\end{array}$ & $23,0 \pm 4,27$ & $19,1 \pm 3,9$ & $17,6 \pm 3,41$ & $18,1 \pm 3,67$ \\
\hline $\begin{array}{l}\text { Grad medio subaórtico } \\
(\mathrm{mmHg})\end{array}$ & $59,0 \pm 27,01$ & $15,6 \pm 11,6$ & $16,8 \pm 12,62$ & $16,4 \pm 6,98$ \\
\hline $\begin{array}{l}\text { Grad máximo subaórtico } \\
(\mathrm{mmHg})\end{array}$ & $80,7 \pm 29,43$ & $19,0 \pm 15,56$ & $14,6 \pm 8,87$ & $13,9 \pm 7,69$ \\
\hline
\end{tabular}

Qx: quirúrgico; Grad: gradiente.

Los datos ecocardiográficos basales de los pacientes en el preoperatorio se situaron en una media de 19,4 $\pm 3,78$ $\mathrm{mm}$ para el grosor del tabique interventricular en diástole, 23,0 \pm 4,27 mm en sístole, 59,0 $\pm 27,01 \mathrm{mmHg}$ para el gradiente medio subaórtico y $80,7 \pm 29,43 \mathrm{mmHg}$ para el gradiente máximo. En el postquirúrgico inmediato (aún intraoperatoriamente) se evidenció una disminución de todos los parámetros con medias de 13,6 \pm 2,11 mm, 19,1 $\pm 3,9 \mathrm{~mm}, 15,6 \pm 11,6 \mathrm{mmHg}(\mathrm{p}<0,01)$ y $19,0 \pm 15,56$ $\mathrm{mmHg}(\mathrm{p}<0,01)$, respectivamente. En el seguimiento al mes, los valores se mantuvieron estables con medias de $13,0 \pm 2,17 \mathrm{~mm}$ para el grosor del tabique interventricular en diástole, 17,6 \pm 3,41 mm en sístole, 16,8 \pm 12,62
$\mathrm{mmHg}$ de gradiente medio subaórtico $(\mathrm{p}<0,01$ en relación al preoperatorio) y $14,6 \pm 8,87 \mathrm{mmHg}$ para el gradiente máximo ( $\mathrm{p}<0,01$ en relación al preoperatorio). $\mathrm{Al}$ año nos encontramos con una media de $12,9 \pm 2,34 \mathrm{~mm}$, $18,1 \pm 3,67$ mm, 16,4 $\pm 6,98 \mathrm{mmHg}(\mathrm{p}<0,01$ en relación al preoperatorio) y $13,9 \pm 7,69 \mathrm{mmHg}(\mathrm{p}<0,01$ en relación al preoperatorio), respectivamente. A destacar que ningún paciente presentó un gradiente subaórtico $\geq 30 \mathrm{mmHg}$ durante el seguimiento.

\section{Seguimiento clínico (Tabla 4).}

\begin{tabular}{|c|c|c|c|}
\hline $\begin{array}{c}\text { Clase funcional } \\
\text { NYHA }\end{array}$ & $\begin{array}{l}\text { Pre quirúrgica } \\
\qquad(\mathrm{N}=52)\end{array}$ & $\begin{array}{l}1 \text { mes } \\
(\mathrm{N}=49)\end{array}$ & $\begin{array}{c}1 \text { año } \\
(\mathrm{N}=42)\end{array}$ \\
\hline I & 0 & 40 & 38 \\
\hline II & 8 & 9 & 4 \\
\hline III & 42 & 0 & 0 \\
\hline IV & 2 & 0 & 0 \\
\hline
\end{tabular}

En el preoperatorio, $8(15,4 \%)$ de los pacientes se encontraban en clase funcional II de la NYHA, 42 (80\%) en clase III, y $2(3,8 \%)$ en clase IV. Al mes del seguimiento 40 $(81,6 \%)$ de los pacientes se encontraban en clase funcional I, y $9(18,4 \%)$ en clase II. Luego del año de seguimiento, $38(90,4 \%)$ se encontraba en clase I, y $4(9,6 \%)$ en clase II. Entre los datos postoperatorios a destacar encontramos un tiempo de estancia en unidad de cuidados intensivos de 5,3 $\pm 9,13$ días y $10,7 \pm 11,97$ días de estancia hospitalaria total. Entre las complicaciones hemodinámicas un 5,8\% de incidencia de bajo gasto cardíaco postoperatorio y $3,8 \%$ de bloqueos $\mathrm{AV}$, todos recuperados espontáneamente sin necesidad de implante de marcapasos definitivos en ningún caso. Como complicaciones infecciosas, un caso de foco respiratorio y otro de foco urinario. Un cuadro confusional y un accidente isquémico transitorio como complicaciones neurológicas, así como un caso de insuficiencia renal aguda que no requirió hemodiálisis. Entre las complicaciones respiratorias, además de la infección, destaca un caso de neumotórax.

\section{Discusión}

Los resultados mostrados demuestran que la intervención puede realizarse de manera segura, sin tasas de compli- 
Resultados a corto y mediano plazo del tratamiento de la obstrucción al tracto de salida del ventrículo...

Paredes F, et al.

Figura 1. Imágenes ecocardiográficas previas y posterior a la intervención

a) Grosor del tabique interventricular previo a la intervención. b) Imagen intraoperatoria posterior a la resección del septo. c) doppler con gradiente subaórtico previo a la intervención. d) doppler con gradiente subaórtico posterior a la resección septal.

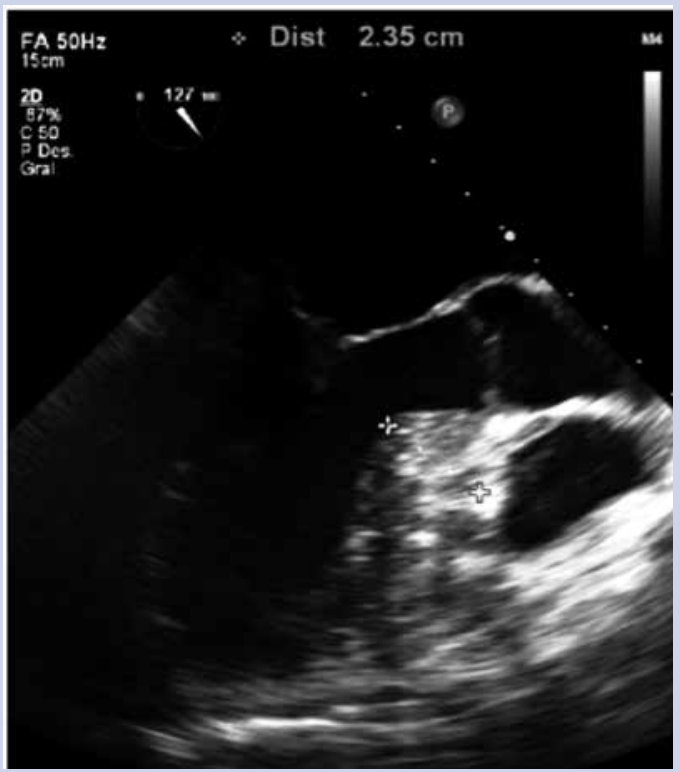

Vmáx: $512 \mathrm{~cm} / \mathrm{s}$

GP máx: $105 \mathrm{mmHg}$

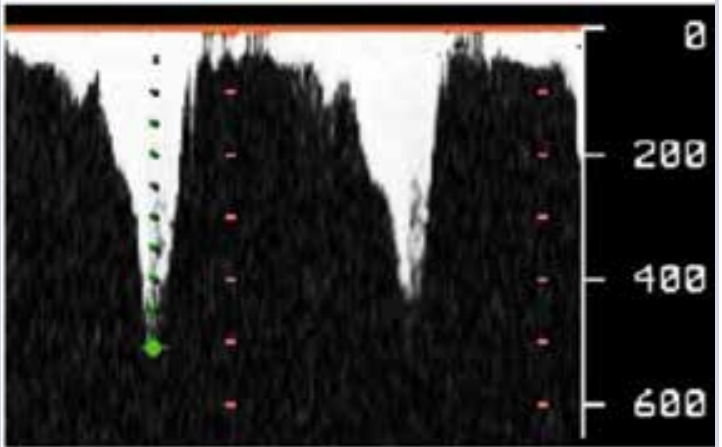

C

A

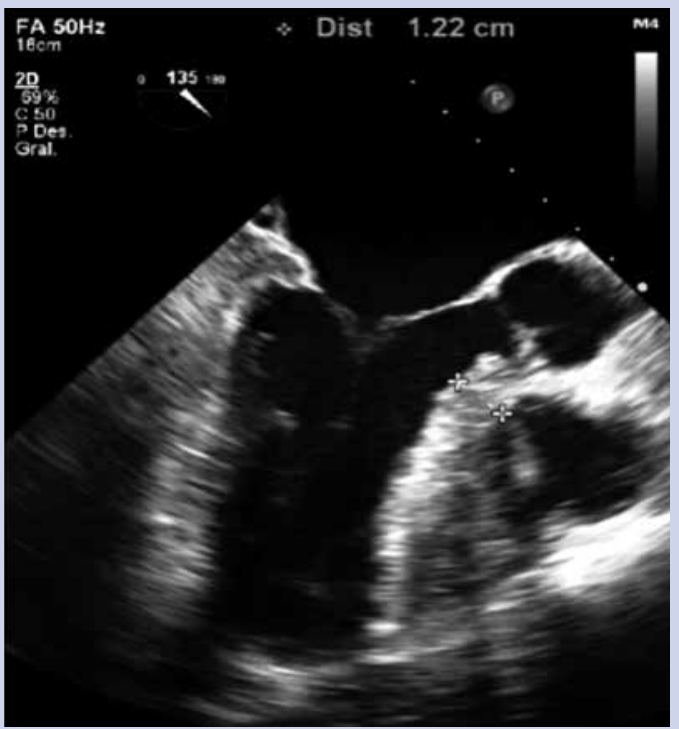

Vmáx: $187 \mathrm{~cm} / \mathrm{s}$

GP máx: $14 \mathrm{mmHg}$

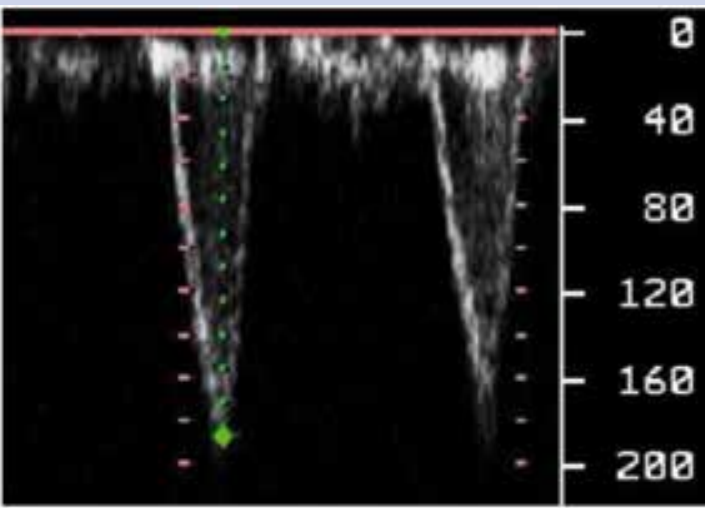

B

D 
caciones mayores a las presentadas en la literatura. ${ }^{20,21}$ Si bien la mortalidad es elevada respecto a la presentada en otros estudios, debemos tener en cuenta que nuestra muestra de pacientes es pequeña, por lo que la aparición aún poco frecuente de un efecto adverso como éste tiene mucha importancia. Sin embargo, ya que una parte importante de nuestra población se encontraba afecta por otras patologías cardíacas concomitantes que fueron resueltas en el mismo acto quirúrgico, podemos considerarlas como factores de riesgo sobreagregado, lo cual podría explicar la mortalidad presentada. Además, de los tres casos, sólo uno de ellos presentaba una miocardiopatía obstructiva aislada y fue sometido a una miectomía videosasistida sin otro procedimiento asociado. Se trataba de un paciente con una cirrosis hepática avanzada y un trastorno basal importante de la coagulación que requirió ser politransfundido con hemoderivados en el postoperatorio lo cual desencadenó un cuadro de distress respiratorio asociado a una sepsis.

La obstrucción al tracto de salida del ventrículo izquierdo es una entidad de etiología variada. Entre las causas más frecuentes figura la estenosis causada por una membrana subaórtica. No está claro si esta anomalía tiene un origen congénito o adquirido ya que ha sido descrita tanto en neonatos como en niños pero nunca en el período prenatal. ${ }^{14}$ Sí hay evidencia de que es una enfermedad progresiva, aunque la progresión puede ser muy variable entre pacientes. ${ }^{1,3,4,14,15}$ Otra causa menos frecuente de OTSVI es la miocardiopatía hipertrófica, una anomalía de origen congénito que provoca una obstrucción tanto mecánica como dinámica al tracto de salida del ventrículo izquierdo.

La cirugía es el "gold estándar" en el tratamiento de pacientes con gradiente elevado y síntomas de insuficiencia cardíaca refractarios al tratamiento médico óptimo. Cuando la obstrucción está causada por la miocardiopatía hipertrófica, la ablación con alcohol del septo podría ser utilizada como opción en pacientes con muy elevado riesgo quirúrgico. ${ }^{8,16}$ La preferencia por el tratamiento quirúrgico es debida a la evidencia existente respecto a los buenos resultados, tanto a corto como mediano plazo, en cuanto a disminución del gradiente, tasa de recidiva y mejoría clínica. 2,17,18,19

Nuestro servicio cuenta con experiencia en el tratamiento de esta patología ya que es Centro de Referencia Nacional para el tratamiento de la miocardiopatía hipertrófica obstruciva. Los resultados mostrados demuestran que la intervención puede realizarse de manera segura, sin tasas de complicaciones mayores a las presentadas en la literatura. $^{20,21}$

Partiendo de la técnica descrita por Morrow en 1978, presentamos algunas modificaciones que consideramos brindan seguridad y efectividad al tratamiento. La adición de la visión directa del tracto de salida del ventrículo izquierdo aportada por la óptica y la miectomía realizada mediante el bisturí de asa consiguen una resección más uniforme y completa, sobre todo en las hipertrofias asimétricas.

Los resultados clínicos y ecocardiográficos presentados durante el seguimiento avalan la efectividad de la técnica ya que ningún paciente presentó un gradiente residual elevado por lo que ninguno precisó ser reintervenido por este motivo. Además, todos se encontraron en clase funcional I-II posterior a la intervención.

En conclusión, la cirugía ha demostrado muy buenos resultados como para ser considerada el tratamiento de elección en la OTSVI. La técnica quirúrgica presentada podría aportar beneficios en cuanto a la facilitar la resección gracias a la mejoría de la visión sobre el tracto de salida.

Al ser una patología poco frecuente, deberían concentrarse los casos en centros con experiencia que se dediquen a esta patología.

\section{Referencias:}

1. YACOUB M, ONUZO O, RIEDEL B, RADLEY-SMITH R. Mobilization of the left and right fibrous trigones for relief of severe left ventricular outflow obstruction. J Thorac Cardiovasc Surg 1999; 117: 126-132.

2. KARAMLOU T, GUROFSKY R, BOJCEVSKI A, WI-
LLIAMS WG, CALDARONE CA, VAN ARSDELL GS, et al: Prevalence and associated risk factors for intervention in 313 children with subaortic stenosis. Ann Thorac Surg 2007; 84: 900-906.

3. PARRY AJ, KOVALCHIN JP, SUDA K, MCELHINNEY DB, WUDEL J, SILVERMAN NH, et al: Resection of subaortic stenosis; can a more agressive approach be justified?. Eur J Car- 
diothorac Surg 1999;15: 631-638.

4. BRAUNER R, LAKS H, DRINKWATER DC JR, SHVARTS O, EGHBALI K, GALINDO A. Benefits of early surgical repair in fixed subaortic stenosis. J Am Coll Cardiol 1997;30:1835-1842.

5. MINAKATA K, DEARANI JA, NISHIMURA RA, MARON BJ, DANIELSON GK. Extended septal myectomy for hypertrophic obstructive cardiomyopathy with anomalous mitral papillary muscles or chordae. J Thorac Cardiovasc Surg 2004;127:481-489.

6. MARON BJ, GARDIN JM, FLACK JM, GIDDING S, KUROSAKI T, BILD D. Prevalence of hypertrophic cardiomyopathy in a general population of Young adults: echocardiographic analysis of 4111 subjects in the CARDIA Study Coronary Artery Risk Development in (Young) Adults. Circulation 1995;92:785-9.

7. MINAKATA K, DEARANI JA, O'LEARY PW, DANIELSON GK. Septal myectomy for obstructive hypertrophic cardiomyopathy in pediatric patients: Early and late results. Ann Thorac Surg 2005;80:1424-1429.

8. MARON BJ, MCKENNA WJ, DANIELSON GK, KAPPENBERGER LJ, KUHN HJ, SEIDMAN CE, ET AL. American College of Cardiology/European Society of Cardiogy clinical expert consensus document on hypertrophic cardiomyopathy. $\mathrm{J}$ Am Coll Cardiol 2003;42:1678-713.

9. MARON BJ. Hypertrophyc cardiomyopathy; a systematic review. JAMA 2002;287:1308-20.

10. MARON BJ, SEIDMAN CE,ACKERMAN MJ, TOWBIN JA, MARON MS, OMMEN SR, et al: How should hypertrophic cardiomyopathy be classified? What's in a name? Dilemmas in nomenclature characterizing hypertrophic cardiomyipathy and left ventricular hypertrophy. Circ. Cardiovasc Genet 2009;2:81-5.

11. GERSH B, BONOW R, FIFER M, NAIDU S, OMMEN S, SEIDMAN C, et al: 2011 ACCF/AHA Guideline for he diagnosis and treatment of hypertrophic cardiomyopathy: Executive summary. Circulation 2011;124;2761-2796.

12. WIGLE ED, SASSON Z, HENDERSON MA, RUDDY TD, FULOP J, RAKOWSKI H, et al: Hypertrophic cardiomyopathy: the importance of the side and the extent of hypertrophy: a re- view. Prog Cardiovasc Dis. 1985;28:1-83.

13. WIGLE ED, RAKOWSKI H, KIMBALL BP, WILLIAMS WG. Hypertrophic cardiomyopathy: clinical spectrum and treatment. Circulation 1995;92:1680-92.

14. SERRAF A, ZOGHBY J, LACOUR-GAYET F, HOUEL R, BELLI E, GALLETTI L, et al: Surgical treatment of subaortic stenosis: A seventeen-year experience. J Thorac Cardiovasc Surg 1999;117:669-678.

15. DODGE-KHATAMI A, SCHMID M, ROUSSON V, FASNACHT M, DOELL C, BAUERSFELD U, et al: Risk factors for reoperation after relief of congenital subaortic stenosis. Eur J Cardiothorac Surg 2008;33:885-889.

16. DEARANI JA, DANIELSON GK. Septal myectomy for hypertrophic cardiomyopathy. Oper Tech Thorac Cardiovas Surg 2004; 9:278-292.

17. SCHULTE HD, BORISOV K, GAMS E, GRAMSCH-ZABEL H, LÖSSE B, SCHWARTZKOPFF B. Management of symtomatic hypertrophic obstructive cardiomyopathy: long-term results after surgical therapy. Thorac Cardiovasc Surg 1999;47:213-218.

18. OMMEN SR, MARON BJ, OLIVOTTO I, MARON MS, CECCHI F, BETOCCHI S, et al: Long-term effects of surgical septal myectomy on survival in patients with obstructive hypertrophic cardiomyopathy. J Am Coll Cardiol 2005;46:470-476.

19. WOO A, WILLIAMS WG, CHOI R, WIGLE ED, ROZENBLYUM E, FEDWICK K, et al: Clinical and echocardiographic determinants of long-term survival after surgical myectomy in obstructive hypertrophic cardiomyopathy. Circulation 2005; 111 : 2033-2041.

20. IACOVONI A, SPIRITO P, SIMON C, LASCONE M, DI DEDDA G, DE FILIPPO P, et al: A contumporary european experience with surgical septal myectomy in hypertrophic cardiomyopathy. European Heart Journal 2012;10:1093.

21. ANAGNOSTOPOULOS P, JOHNSON N, ROBERTSON L, SAPRU A, AZAKIE A. Surgical management of left ventricular outflow tract obstruction. J Card Surg 2012;27:103-111. 